From the Ospedali Riuniti di Bergamo, Bergamo; University of Pavia, Istituto Di Ricovero e Cura a Carattere Scientifico Policlinico San Matteo, Pavia; S. Bortolo Hospital, Vicenza; University of Padua, Padua; University of Florence, Florence, Italy; University of Cologne, Cologne, Germany; Medical University of Vienna, Vienna, Austria; and Mayo Clinic, Rochester, MN

Submitted December 27, 2010: accepted March 14, 2011; published online ahead of print at www.jco.org on July 11, 2011.

Supported by the Associazione Italiana per la Ricerca sul Cancro (AIRC to A.M.V.) and by the Special Program Molecular Clinical Oncology 51000 (to AIRC Gruppo Italiano Malattie Mieloproliferative)

T.B., J.T., F.P., and A.T. contributed equally to this work.

Authors' disclosures of potential conflicts of interest and author contributions are found at the end of this article.

Corresponding author: Ayalew Tefferi, MD, Mayo Clinic, 200 First St SW Rochester, MN 55905; e-mail: tefferi.ayalew@mayo.edu.

(C) 2011 by American Society of Clinica Oncology

0732-183X/11/2923-3179/\$20.00

DOI: 10.1200/JCO.2010.34.5298

\title{
Survival and Disease Progression in Essential Thrombocythemia Are Significantly Influenced by Accurate Morphologic Diagnosis: An International Study
}

Tiziano Barbui, Juergen Thiele, Francesco Passamonti, Elisa Rumi, Emanuela Boveri, Marco Ruggeri, Francesco Rodeghiero, Emanuele S.G. d'Amore, Maria Luigia Randi, Irene Bertozzi, Filippo Marino, Alessandro M. Vannucchi, Elisabetta Antonioli, Valentina Carrai, Heinz Gisslinger, Veronika Buxhofer-Ausch, Leonhard Müllauer, Alessandra Carobbio, Andrea Gianatti, Naseema Gangat, Curtis A. Hanson, and Ayalew Tefferi

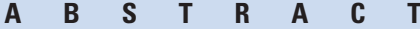

\section{Purpose}

The WHO diagnostic criteria underscore the role of bone marrow (BM) morphology in distinguishing essential thrombocythemia (ET) from early/prefibrotic primary myelofibrosis (PMF). This study examined the clinical relevance of such a distinction.

\section{Methods}

Representatives from seven international centers of excellence for myeloproliferative neoplasms convened to create a clinicopathologic database of patients previously diagnosed as having ET $(N=1,104)$. Study eligibility criteria included availability of treatment-naive BM specimens obtained within 1 year of diagnosis. All bone marrows subsequently underwent a central re-review.

\section{Results}

Diagnosis was confirmed as ET in 891 patients (81\%) and was revised to early/prefibrotic PMF in $180(16 \%)$; 33 patients were not evaluable. In early/prefibrotic PMF compared with ET, the 10 -year survival rates (76\% and $89 \%$, respectively) and 15 -year survival rates (59\% and $80 \%$, respectively), leukemic transformation rates at 10 years (5.8\% and $0.7 \%$, respectively) and 15 years $(11.7 \%$ and $2.1 \%$, respectively), and rates of progression to overt myelofibrosis at 10 years $(12.3 \%$ and $0.8 \%$, respectively) and 15 years $(16.9 \%$ and $9.3 \%)$ were significantly worse. The respective death, leukemia, and overt myelofibrosis incidence rates per 100 patient-years for early/prefibrotic PMF compared with ET were $2.7 \%$ and $1.3 \%$ (relative risk $[R R], 2.1 ; P<.001), 0.6 \%$ and $0.1 \%(R R, 5.2 ; P=.001)$, and $1 \%$ and $0.5 \%(R R, 2.0 ; P=.04)$. Multivariable analysis confirmed these findings and also identified age older than 60 years (hazard ratio $[H R], 6.7)$, leukocyte count greater than $11 \times 10^{9} / \mathrm{L}(H R, 2.01)$, anemia $(H R, 2.95)$, and thrombosis history (HR, 2.81) as additional risk factors for survival. Thrombosis and JAK2V617F incidence rates were similar between the two groups. Survival in ET was similar to the sex- and age-standardized European population.

\section{Conclusion}

This study validates the clinical relevance of strict adherence to WHO criteria in the diagnosis of ET and provides important information on survival, disease complication rates, and prognostic factors in strictly WHO-defined ET and early/prefibrotic PMF.

\section{J Clin Oncol 29:3179-3184. (C) 2011 by American Society of Clinical Oncology}

\section{INTRODUCTION}

The differential diagnosis of increased platelet count includes reactive thrombocytosis, essential thrombocythemia (ET), chronic myelogenous leukemia, polycythemia vera (PV), primary myelofibrosis (PMF), refractory anemia with ring sideroblasts and marked thrombocytosis, and other chronic my- eloid neoplasms. ${ }^{1}$ Accurate diagnosis is important in terms of both prognostication and treatment. The WHO classification system for hematopoietic tumors provides clear and concise guidelines in distinguishing ET from both reactive thrombocytosis and other myeloid neoplasms. ${ }^{1}$ Accordingly, the presence of a clonal marker (eg, JAK2 or MPL mutation), which is present in the majority of patients 
with ET, PV or PMF, reliably excludes the possibility of reactive thrombocytosis, whereas chronic myelogenous leukemia and refractory anemia with ring sideroblasts and marked thrombocytosis are expected to be invariably associated with $B C R-A B L 1$ and dyserythropoiesis, respectively. ${ }^{2}$ It should be noted, however, that $J A K 2 \mathrm{~V} 617 \mathrm{~F}$, which is the most frequent mutation in BCR-ABL1negative myeloproliferative neoplasms (MPNs), has limited discriminatory value. ${ }^{3,4}$ Bone marrow (BM) histology, including megakaryocyte morphology and degree of myeloproliferation, is key in distinguishing ET from the prodromal stages of PV and PMF. ${ }^{5}$ Overt BM fibrosis might be absent in early/prefibrotic PMF, leading to a spurious diagnosis of ET if one does not pay attention to BM morphology. This study was performed under the auspices of the International Working Group for MPN Research and Treatment; the main objective of the study was to address the prognostic relevance of distinguishing early/prefibrotic PMF associated with thrombocytosis from ET.

\section{METHODS}

Physician scientists and hematopathologists from seven international centers of excellence for MPNs convened to create a clinicopathologic database of patients who were diagnosed and treated for ET in their respective locales. The study was approved by the institutional review board of each institution. Study eligibility criteria included the availability of representative, treatment-naive $\mathrm{BM}$ biopsies obtained at diagnosis or within 1 year of diagnosis. Diagnostic criteria used were those in effect at the time of initial diagnosis; in most instances, the Polycythemia Vera Study Group criteria ${ }^{6}$ were used for diagnoses made before 2002, and the WHO criteria were used for diagnoses made thereafter. ${ }^{7,8}$ However, before central re-review, the local hematopathologists from each study center were given the opportunity to revise the diagnoses from their older BM slides (hematoxylin-eosin staining and silver impregnation after Gomori or Gordon-Sweet) according to the updated 2008 WHO criteria. $^{8}$

All BM biopsies subsequently underwent a central re-review by one of the authors (J.T.), who is a coinvestigator in this project as well as the author of the WHO chapter on diagnostic criteria for PMF, PV, and ET. ${ }^{8}$ The final diagnostic assignment was made according to the results of the central rereview. In the course of this review, corresponding guidelines to recognize characteristic histologic BM patterns, including fiber scoring, ${ }^{9}$ were applied to identify occurrences of early/prefibrotic PMF that were spuriously diagnosed as ET. Both local pathologists and the expert reviewer had access to patient information that is necessary for routine interpretation of BM morphology. However, the review process was completely blinded to outcome data, which were analyzed after the completion of the histopathology review. The diagnosis of post-ET myelofibrosis (or progression into overt myelofibrosis) was made by corresponding clinical features, including worsening anemia, splenomegaly, leukoerythroblastosis, or anisopoikilocytosis with teardrop erythrocytes. Leukemic transformation met criteria for acute myeloid leukemia per the WHO system. ${ }^{7,8}$ Allele-specific polymerase chain reaction techniques were utilized to screen for JAK2V617F in each participating center. ${ }^{10-12}$

A total of 116 disease-relevant parameters were extracted in each occurrence; these parameters included dates of diagnosis, death, leukemic transformation, progression into overt myelofibrosis, and vascular events. All statistical analyses considered parameters at the time of initial diagnosis. Differences in the distribution of continuous variables between categories were analyzed by the Mann-Whitney test. Patient groups with nominal variables were compared by the $\chi^{2}$ test. Outcomes of interest were reported as rates per 100 patient-years as well as by cumulative incidences calculated at 5, 10, and 15 years from the date of diagnosis. Incidence rate ratios between groups were estimated and tested. Overall survival analysis was considered from the date of diagnosis to date of death (uncensored) or last contact (censored). Leukemiafree, overt myelofibrosis-free, and thrombosis-free survivals were calculated from the date of diagnosis to date of leukemic transformation, progression into overt myelofibrosis, and thrombotic complication (all uncensored), respectively, or last contact/date of death (all censored). Survival estimates patients with ET and early-PMF were compared with those obtained by applying to the population the 2008 Eurostat age- and sex-standardized incidence rates for all causes of death, by using the indirect standardization procedure. All survival curves were prepared by the Kaplan-Meier method and were compared by the log-rank test. The Cox proportional hazard regression model was used for multivariable analysis. $P$ values less than .05 were considered significant.

\section{RESULTS}

A total of 1,104 patients met minimal criteria for study inclusion: a local diagnosis of ET with pretreatment BM obtained at time of diagnosis or within 1 year of diagnosis. All occurrences were diagnosed between 1975 and 2008 and were recruited from Pavia, Italy ( $\mathrm{n}=$ $271)$, Bergamo, Italy $(\mathrm{n}=186)$, Vicenza, Italy $(\mathrm{n}=152)$, Rochester, $\mathrm{MN}(\mathrm{n}=130)$, Padua, Italy $(\mathrm{n}=123)$, Vienna, Austria $(\mathrm{n}=122)$, and Florence, Italy $(\mathrm{n}=120)$. Central re-review led to a diagnosis of

Table 1. Comparison of Patient Characteristics at Presentation in ET Versus Early/Prefibrotic PMF

\begin{tabular}{|c|c|c|c|}
\hline Characteristic & ET $(n=891)$ & $\begin{array}{l}\text { Early/Prefibrotic } \\
\text { PMF }(n=180)\end{array}$ & $P$ \\
\hline Age, years & & & .66 \\
\hline Median & 56 & 57 & \\
\hline Range & $13-91$ & $21-88$ & \\
\hline \multicolumn{4}{|l|}{ Sex } \\
\hline Male & 370 & 74 & \\
\hline Female & 521 & 106 & .92 \\
\hline Leukocyte count, $\times 10^{9} / \mathrm{L}$ & & & $<.001$ \\
\hline Median & 8.6 & 9.7 & \\
\hline Percentile range, 5 th to 95 th & $5.3-14.2$ & $6.9-17.0$ & \\
\hline Hemoglobin, g/dL & & & .01 \\
\hline Median & 14.1 & 13.8 & \\
\hline Percentile range, 5 th to 95 th & $11.9-16.1$ & $11.4-15.8$ & \\
\hline Platelets, $\times 10^{9} / \mathrm{L}$ & & & .002 \\
\hline Median & 774 & 902 & \\
\hline Percentile range, 5th to 95th & $500-1,464$ & $564-1,898$ & \\
\hline Lactate dehydrogenase, $\mathrm{mU} / \mathrm{mL}^{*}$ & & & $<.001$ \\
\hline Median & 298 & 429 & \\
\hline Percentile range, 5th to 95th & $150-582$ & $121-875$ & \\
\hline Palpable splenomegaly & & & .04 \\
\hline No. & 146 & 41 & \\
\hline$\%$ & 16 & 23 & \\
\hline Circulating $\mathrm{CD} 34^{+}$cell count/ $\mu \mathrm{L} \dagger$ & & & .03 \\
\hline Median & 2 & 4.7 & \\
\hline Range & $0-15.2$ & $0-60$ & \\
\hline JAK2V617F positiveł & & & .56 \\
\hline No. & 422 & 67 & \\
\hline$\%$ & 61 & 58 & \\
\hline Reticulin fibrosis grade $1^{9}$ & & & $<.001$ \\
\hline No. & 24 & 43 & \\
\hline$\%$ & 3 & 24 & \\
\hline
\end{tabular}

Abbreviations: ET, essential thrombocythemia; PMF, primary myelofibrosis.

*No. of patients $=519$.

†No. of patients $=246$

$\ddagger$ No. of patients $=805$ 
WHO-defined ET in 891 instances (81\%) and early/prefibrotic PMF in $180(16 \%)$; the remaining $33(3 \%) \mathrm{BM}$ specimens were either qualitatively inadequate for accurate classification or represented reactive occurrences. The concordance rate for the diagnosis of ET between the seven local pathologists and the referee pathologist was $81 \%$ when considering the total study cohort, including nonrepresentative biopsy samples, and was $83 \%$ for discriminating ET from early/ prefibrotic PMF.

\section{Patient Characteristics at Presentation}

Table 1 provides a comparative illustration of presenting clinical and laboratory features for 891 patients with ET and 180 patients with early/prefibrotic PMF. Significant differences were seen for leukocyte count (greater in early/prefibrotic PMF than ET), hemoglobin level (greater in ET than early/prefibrotic PMF), platelet count (greater in early/prefibrotic PMF than ET), serum lactate dehydrogenase (LDH) level (greater in early/prefibrotic PMF than ET), circulating CD34 cell count (greater in early/prefibrotic PMF than ET), incidence of palpable splenomegaly (greater in early/prefibrotic PMF than ET), and frequency of grade $1 \mathrm{BM}$ fibrosis (greater in early/prefibrotic PMF than ET). Of note, age and sex distribution and JAK2V617F mutational frequencies were similar between the two groups.

\section{Clinical Course of Patients With ET Versus Early/Prefibrotic PMF}

Median follow-up from time of diagnosis was 6.2 years (range, 0 to 27 years) for ET and 7 years (range, 0 to 27.2 years) for early/ prefibrotic $\operatorname{PMF}(P=.3)$. During this period, 87 patients $(10 \%)$ with ET and $40(22 \%)$ with early/prefibrotic PMF had died $(P<.001)$. Cytoreductive therapy was instituted in 507 patients (57\%) with ET and in $123(68 \%)$ with early/prefibrotic PMF $(P=.005)$. In total, leukemic and overt fibrotic transformations were documented in eight patients (1\%) and 32 patients (4\%), respectively, with ET and in nine patients $(5 \%)$ and 14 patients (8\%), respectively, with early/ prefibrotic $\operatorname{PMF}(P=.0012$ for leukemic transformation; $P=.04$ for overt fibrotic transformations; Table 2). In contrast, the incidences of thrombotic complications were similar between the two groups: 109 $(12 \%)$ in ET and $26(15 \%)$ in early/prefibrotic PMF $(P=.51)$. The 15 -year cumulative risks of death, leukemic transformation, overt fibrotic progression, and thrombotic complications were approximately $25 \%, 2 \%, 9 \%$, and $22 \%$, respectively, for ET and were $56 \%$, $12 \%, 17 \%$, and $25 \%$, respectively, for early/prefibrotic PMF (Table 2). Overall (Fig 1A; $P<.001$ ), leukemia-free (Fig 1B; $P<.001$ ) and overt myelofibrosis-free (Fig $1 \mathrm{C} ; P=.03$ ) survivals were significantly better in ET than in early/prefibrotic PMF, whereas thrombosis-free survival was similar in the two groups (Fig 1D). Median overall survival was 11.2 years for early/prefibrotic PMF and was 14.7 years ET $(P<.001)$. Survival in ET was similar to that of the sex- and age-standardized European population (Fig 2).

\section{Multivariable Analysis of Overall and Event-Free Survival}

Multivariable analysis was performed on data from all 1,071 study patients by using Cox regression model and included several covariates with potential prognostic relevance: BM histopathology (ie, ET $v$ early/prefibrotic PMF), age (older than 60 years), sex, history of thrombosis, leukocytosis $\left(>11 \times 10^{9} / \mathrm{L}\right)$, anemia, extreme thrombocytosis (platelet count $>1,000 \times 10^{9} / \mathrm{L}$ ), JAK2V617F, and grade $1 \mathrm{BM}$ reticulin fibrosis (Table 3 ). Of note, the threshold level of 11,000 $\times$ $10^{9} / \mathrm{L}$ leukocytes was chosen on the basis of receiver operating characteristic (ROC) analysis.

The prognostic relevance of BM histopathology remained significant for survival $(P=.03)$, leukemic transformation $(P=.007)$ and overt fibrotic progression $(P=.019)$. In addition, survival was independently and negatively affected by older age (age older than 60 years; $P<.001$ ), leukocytosis (leukocyte count $>11 \times 10^{9} / \mathrm{L} ; P<.001$ ), anemia (hemoglobin $<12 \mathrm{~g} / \mathrm{dL} ; P<.001$ ) and history of thrombosis $(P<.001)$. BM histopathology-independent risk factors for overt fibrotic progression included older age, anemia, and, interestingly, absence of JAK2V617F (Table 3). The corresponding risk factors for leukemic transformation were history of thrombosis and extreme thrombocytosis (Table 3).

\section{DISCUSSION}

$B C R-A B L 1$ and JAK2V617F mutation screening have helped overcome some of the diagnostic uncertainties in ET. ${ }^{2}$ However, the molecular and phenotypic similarities between ET and early/prefibrotic

\begin{tabular}{|c|c|c|c|c|c|c|c|c|}
\hline Event & $\begin{array}{l}\text { No. of } \\
\text { Events }\end{array}$ & $\begin{array}{l}\% \text { of } \\
\text { Events }\end{array}$ & $\begin{array}{c}\text { Incidence per } \\
100 \text { Patient-Years }\end{array}$ & IRR & $P$ & $\begin{array}{l}\text { 5-Year Cumulative } \\
\text { Incidence (\%) }\end{array}$ & $\begin{array}{l}\text { 10-Year Cumulative } \\
\text { Incidence (\%) }\end{array}$ & $\begin{array}{l}\text { 15-Year Cumulative } \\
\text { Incidence }(\%)\end{array}$ \\
\hline \multicolumn{9}{|l|}{ Thrombosis } \\
\hline ET & 109 & 12 & 1.7 & 1.1 & .57 & 8.7 & 16.2 & 21.5 \\
\hline Early/prefibrotic PMF & 26 & 15 & 1.9 & & & 6.6 & 17.9 & 25.4 \\
\hline \multicolumn{9}{|l|}{$\begin{array}{l}\text { Transformation to overt } \\
\text { myelofibrosis }\end{array}$} \\
\hline ET & 32 & 4 & 0.5 & 2.0 & .04 & 0.2 & 0.8 & 9.3 \\
\hline Early/prefibrotic PMF & 14 & 8 & 1 & & & 2.3 & 12.3 & 16.9 \\
\hline \multicolumn{9}{|l|}{ Leukemic transformation } \\
\hline ET & 8 & 1 & 0.1 & 5.2 & .0012 & 0.2 & 0.7 & 2.1 \\
\hline Early/prefibrotic PMF & 9 & 5 & 0.6 & & & 1.5 & 5.8 & 11.7 \\
\hline \multicolumn{9}{|l|}{ Death } \\
\hline ET & 87 & 10 & 1.3 & 2.1 & .0002 & 3.0 & 14.8 & 24.6 \\
\hline Early/prefibrotic PMF & 40 & 22 & 2.7 & & & 8.6 & 24.4 & 56.1 \\
\hline
\end{tabular}

Abbreviations: ET, essential thrombocythemia; PMF, primary myelofibrosis; IRR, incidence rate ratio. 

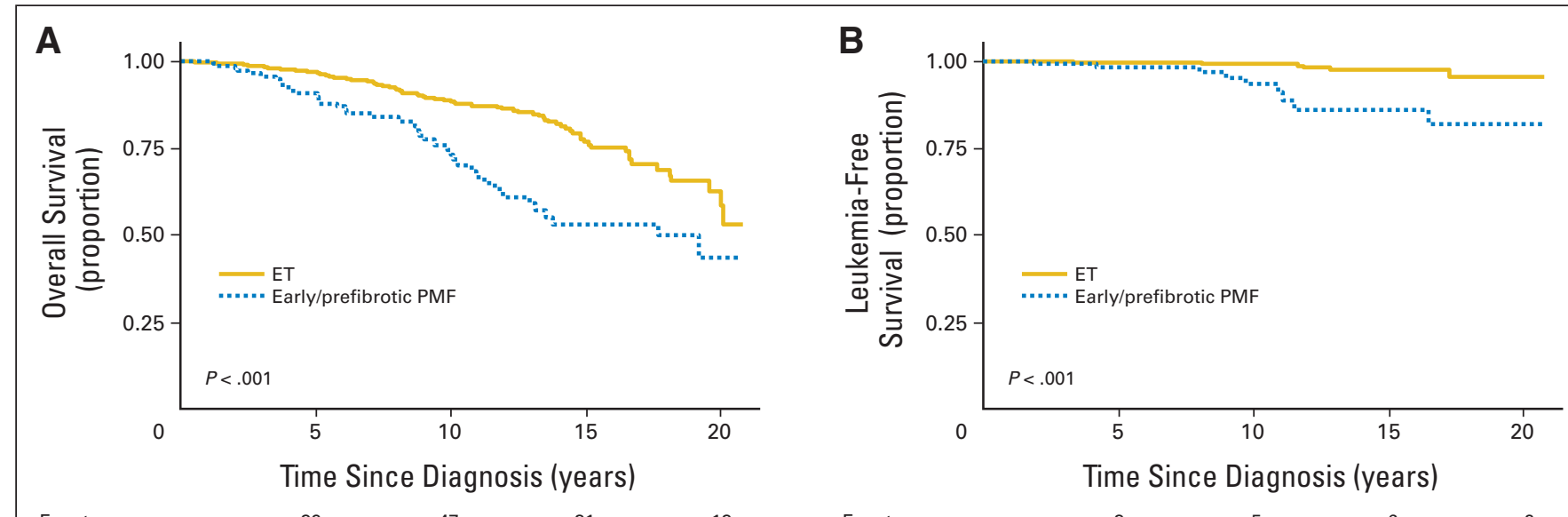

$\begin{array}{lrrrr}\text { Events } & 32 & 47 & 31 & 13 \\ \text { No. at risk } & 628 & 326 & 157 & 57\end{array}$

$\begin{array}{lrrrr}\text { Events } & 3 & 5 & 6 & 2 \\ \text { No. at risk } & 627 & 325 & 156 & 57\end{array}$
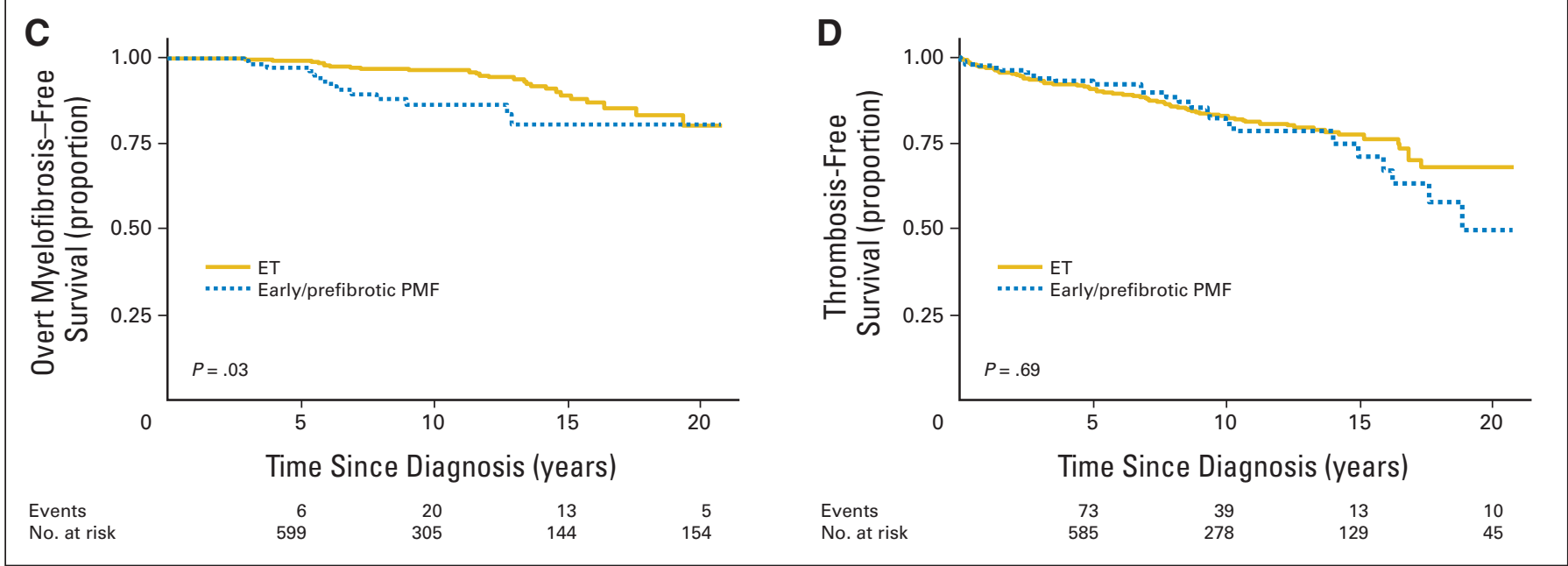

Fig 1. (A) Overall, (B) leukemia-free, (C) overt myelofibrosis-free, and (D) thrombosis-free survivals of patients with true essential thrombocythemia (ET; $n=891$ ) versus early/prefibrotic primary myelofibrosis (PMF; $n=180$ ).

PMF have led to significant controversies and speculations regarding the value of morphology in distinguishing between the two entities and the clinical relevance of such an exercise. ${ }^{13-15}$ In this regard, it should be noted that, independent from the Cologne group who were

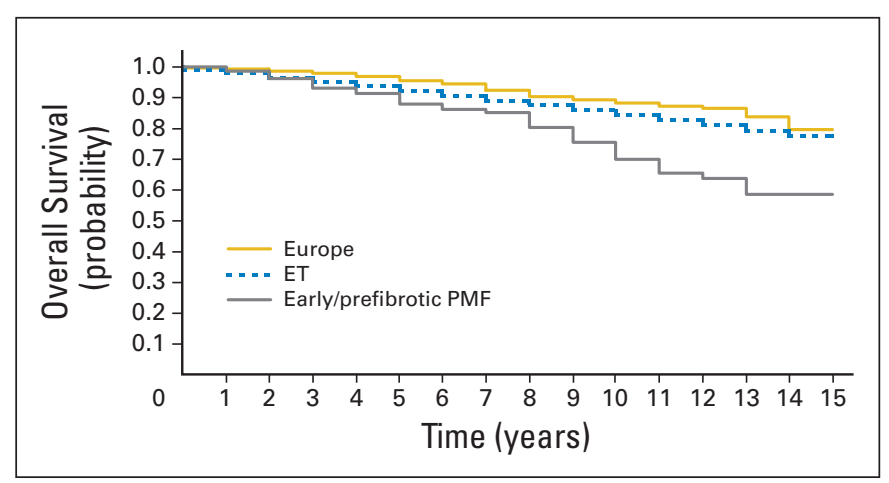

Fig 2. Survival estimates for patients with essential thrombocythemia (ET) and early/prefibrotic primary myelofibrosis (PMF) were compared with those obtained by applying to the population the 2008 Eurostat age- and sexstandardized incidence rates for all causes of death, by using the indirect standardization procedure. instrumental in the original description of the key histologic differences between ET and early/prefibrotic PMF, ${ }^{5}$ several other investigators had endorsed the reliability of BM morphologic and histopathologic characteristics proposed by the WHO. ${ }^{16-19}$ On the other hand, two other groups have raised the issue of reproducibility in this matter. ${ }^{14,15}$ The study by the English group ${ }^{14}$ included a semiquantitative evaluation of approximately 16 BM parameters in 370 biopsy specimens derived from the United Kingdom-PT1 trial ${ }^{20}$ and is, unfortunately, impaired by a number of inconsistencies in design and performance that have been already discussed elsewhere. ${ }^{5}$ In the other study by a French group, ${ }^{15}$ the authors claimed to have applied the WHO classification for ET but described the presence of a minor to moderate reticulin fibrosis in 54\% of their BM biopsies obtained up to 3 years after diagnosis. This is inconsistent with what is expected if one were to strictly adhere to WHO diagnostic criteria, in which minor reticulin fibrosis is much less frequent (Table 1). ${ }^{13}$ Regardless, the more than $80 \%$ concordance rate between local pathologists and the referee pathologist in this study compares favorably with other reported concordance rates related to the diagnosis of histologically complex hematopoietic disorders. ${ }^{21,22}$ Furthermore, our observation that $16 \%$ of ET occurrences recruited from several MPN centers of excellence represented misdiagnosed early/prefibrotic PMF is partly explained by 
Table 3. Results of Cox Multivariable Analysis of Relevant Clinical and Laboratory Parameters on Rates of Death or Progression to Leukemia or Overt Myelofibrosis

\begin{tabular}{|c|c|c|c|c|c|c|c|c|c|}
\hline \multirow[b]{2}{*}{ Parameter at Diagnosis } & \multicolumn{3}{|c|}{ Progression to Myelofibrosis } & \multicolumn{3}{|c|}{ Leukemic Transformation } & \multicolumn{3}{|c|}{ Death } \\
\hline & $H R$ & $95 \% \mathrm{Cl}$ & $P$ & $H R$ & $95 \% \mathrm{Cl}$ & $P$ & $H R$ & $95 \% \mathrm{Cl}$ & $P$ \\
\hline BM histology: early/prefibrotic PMF $v$ ET & 2.35 & 1.15 to 4.80 & .019 & 4.50 & 1.52 to 13.3 & .007 & 1.60 & 1.05 to 2.44 & .030 \\
\hline Age $>60$ years & 2.34 & 1.28 to 4.30 & .006 & 1.86 & 0.65 to 5.29 & .246 & 6.70 & 4.34 to 10.3 & $<.0001$ \\
\hline Previous thrombosis & 1.14 & 0.49 to 2.61 & .767 & 4.09 & 1.40 to 11.9 & .010 & 2.81 & 1.95 to 4.06 & $<.0001$ \\
\hline Male sex & 1.68 & 0.91 to 3.05 & .097 & 1.61 & 0.59 to 4.38 & .350 & 1.30 & 0.91 to 1.86 & .151 \\
\hline Leukocyte count $>11 \times 10^{9} / \mathrm{L}$ & 0.90 & 0.42 to 1.93 & .781 & 0.39 & 0.12 to 1.30 & .125 & 2.01 & 1.39 to 2.90 & .0002 \\
\hline Hemoglobin $<12$ g/dL & 4.22 & 1.76 to 10.1 & .001 & 2.64 & 0.49 to 12.4 & .274 & 2.95 & 1.73 to 5.04 & $<.0001$ \\
\hline Platelets $>1,000 \times 10^{9} / \mathrm{L}$ & 0.60 & 0.30 to 1.23 & .165 & 2.97 & 1.03 to 8.56 & .044 & 1.30 & 0.90 to 1.90 & .167 \\
\hline JAK2V617F presence & 0.37 & 0.17 to 0.79 & .015 & 1.45 & 0.23 to 9.17 & .693 & 1.48 & 0.80 to 2.76 & .216 \\
\hline Reticulin fibrosis grade $1^{9}$ & 0.94 & 0.30 to 2.95 & .922 & 1.63 & 0.41 to 6.5 & .49 & 1.06 & 0.57 to 1.98 & .853 \\
\hline
\end{tabular}

Abbreviations: HR, hazard ratio; BM, bone marrow; PMF, primary myelofibrosis; ET, essential thrombocythemia.

the inclusion of patients diagnosed before 2002, when the now-defunct Polycythemia Vera Study Group diagnostic criteria ${ }^{6}$ were utilized; the latter did not emphasize morphologic scrutiny and instead relied mostly on extent of BM fibrosis to distinguish ET from PMF. ${ }^{23}$

The main objective of this study was to address the clinical relevance of distinguishing WHO-defined ET from early/prefibrotic PMF by subjecting a large number of ET occurrences to a central review of BM morphology and subsequently by comparing clinical presentation and outcome data between confirmed ET occurrences and those patients whose diagnoses were revised to early/prefibrotic PMF. Accordingly, the latter group is not precisely representative of early/ prefibrotic PMF, and this study design may not provide definitive conclusions about differences in the evolution of ET and early/prefibrotic PMF. Regardless, the results of this study indicate that the distinction between ET and early/prefibrotic PMF is not trivial and that accurate diagnosis is prognostically relevant. At the same time, one should note that, although inferior to that seen in patients with ET, event-free survival was still relatively long in patients with early/ prefibrotic PMF. This study also provides laboratory clues for considering the possibility of early/prefibrotic PMF: elevated serum LDH, anemia, leukocytosis, and increased circulating $\mathrm{CD}^{+}{ }^{+}$cell count. Accordingly, the appearance of such characteristics during the clinical course of ET might be considered as an indication for repeating BM examination to entertain the possibility of overt fibrotic transformation. JAK2V617F mutational frequencies were similar between ET and early/prefibrotic PMF, although another recent study had suggested a higher mutant allele burden in the latter. ${ }^{24}$

Given the more aggressive disease biology in non-ET myeloid neoplasms, which might mimic ET in their clinical presentations, it was our hypothesis that survival and disease complication rates in strictly WHO-defined ET would be even more favorable than currently assumed. The results of this study support this contention by revealing a less than 1\% 10-year risk of leukemic or overt fibrotic transformation in morphologically accurate ET. These figures are notably lower than those previously reported in large retrospective studies from independent groups of investigators. For example, in a recent population-based French study of 311 patients with ET, the 10 -year risk of leukemic transformation was reported as $8.3 \%^{25}$; in another multicenter study of 211 patients from China, the 10 -year risk of overt fibrotic transformation was reported as $9.7 \%,{ }^{26}$ and the corresponding figure from a Spanish series was $8.3 \% .{ }^{27}$ Therefore, it is reasonable to assume that data from previous natural history studies in ET are confounded by the inadvertent inclusion of occurrences with early/prefibrotic PMF and do not necessarily apply to patients whose diagnoses are based on strict morphologic criteria. In instances for which an attempt was made to re-review BM histopathology according to WHO criteria, reported disease complication rates were closer to those from this study ${ }^{28,29}: 10$-year risk of leukemic and overt fibrotic transformation rates were $1.4 \%$ and $3.8 \%$, respectively, in one such study from the Mayo Clinic ${ }^{29}$ and were $2.6 \%$ and $3.9 \%$, respectively, in another study from Pavia, Italy. ${ }^{28}$

Multivariable analyses in this study confirmed the prognostic relevance of BM histopathology for survival, leukemic transformation, and overt fibrotic progression. BM histopathology-independent risk factors for survival included older age (older than 60 years), leukocytosis (leukocyte count $>11 \times 10^{9} / \mathrm{L}$ ), anemia (hemoglobin $<12 \mathrm{~g} / \mathrm{dL}$ ), and thrombosis history. Not surprisingly, all four of these risk factors were previously cited in the same capacity, although inconsistently, by other studies. ${ }^{25,26,28-31}$ Some of these previous studies had also reported a similar adverse survival effect from tobacco use, diabetes mellitus, and male sex. In this study, older age and anemia were also shown to be independent risk factors for overt fibrotic progression, and thrombosis history and extreme thrombocytosis were independent risk factors for leukemic transformation. In previous studies, risk factors for leukemic transformation had included leukocytosis, extreme thrombocytosis, and older age $e^{28,30}$; risk factors for overt fibrotic progression included anemia, increased serum LDH, increased reticulin fibrosis, and male sex. ${ }^{28,32}$ Taken together, it is reasonable to conclude that some of the aforementioned risk factors (eg, anemia, increased serum $\mathrm{LDH}$, leukocytosis, extreme thrombocytosis) might be markers of occult early/prefibrotic PMF, and that could explain their association with inferior survival or higher risk of disease progression.

The observations from this study constitute good news for patients with strictly WHO-defined ET; their prognoses might be even better than previously assumed (Fig 2). Of note, the presence of $J A K 2 \mathrm{~V} 617 \mathrm{~F}$ in this study was not detrimental to survival or leukemic transformation and was instead associated with a lower risk of overt fibrotic progression. This study signifies the importance of excluding early/prefibrotic PMF in all patients with ET, especially in the presence of proven surrogate markers, such as characteristic BM morphology, elevated serum $\mathrm{LDH}$, anemia, or leukocytosis. In this regard, the issue is not as much about reproducibility of morphologic interpretation 
but more about increased awareness and effective integration of clinical and laboratory data to make an accurate working diagnosis.

\section{AUTHORS' DISCLOSURES OF POTENTIAL CONFLICTS} OF INTEREST

The author(s) indicated no potential conflicts of interest.

\section{AUTHOR CONTRIBUTIONS}

Conception and design: Tiziano Barbui, Juergen Thiele, Francesco Passamonti, Heinz Gisslinger, Ayalew Tefferi
Administrative support: Tiziano Barbui, Juergen Thiele, Ayalew Tefferi Provision of study materials or patients: Tiziano Barbui, Juergen Thiele, Francesco Passamonti, Ayalew Tefferi

Collection and assembly of data: Tiziano Barbui, Juergen Thiele, Francesco Passamonti, Elisa Rumi, Emanuela Boveri, Marco Ruggeri, Francesco Rodeghiero, Emanuele S.G. d'Amore, Maria Luigia Randi, Irene Bertozzi, Filippo Marino, Alessandro M. Vannucchi, Elisabetta Antonioli, Valentina Carrai, Heinz Gisslinger, Veronika Buxhofer-Ausch, Leonhard Müllauer, Alessandra Carobbio, Andrea Gianatti, Naseema Gangat, Curtis A. Hanson, Ayalew Tefferi

Data analysis and interpretation: Tiziano Barbui, Juergen Thiele, Francesco Passamonti, Alessandra Carobbio, Ayalew Tefferi

Manuscript writing: All authors Final approval of manuscript: All authors

\section{REFERENCES}

1. Vardiman JW, Thiele J, Arber DA, et al: The 2008 revision of the World Health Organization (WHO) classification of myeloid neoplasms and acute leukemia: Rationale and important changes. Blood 114:937951, 2009

2. Tefferi A, Vardiman JW: Classification and diagnosis of myeloproliferative neoplasms: The 2008 World Health Organization criteria and point-of-care diagnostic algorithms. Leukemia 22:14-22, 2008

3. Vannucchi AM, Antonioli E, Guglielmelli $P$, et al: Clinical correlates of JAK2V617F presence or allele burden in myeloproliferative neoplasms: A critical reappraisal. Leukemia 22:1299-1307, 2008

4. Malcovati L, Della Porta MG, Pietra D, et al: Molecular and clinical features of refractory anemia with ringed sideroblasts associated with marked thrombocytosis. Blood 114:3538-3545, 2009

5. Kvasnicka HM, Thiele J: Prodromal myeloproliferative neoplasms: The 2008 WHO classification. Am J Hematol 85:62-69, 2010

6. Murphy $S$, Peterson $P$, Iland $H$, et al: Experience of the Polycythemia Vera Study Group with essential thrombocythemia: A final report on diagnostic criteria, survival, and leukemic transition by treatment. Semin Hematol 34:29-39, 1997

7. Jaffe ES, Harris NL, Stein $\mathrm{H}$, et al: World Health Organization Classification of Tumours of Hematopoietic and Lymphoid Tissues. Lyon, France, IARC Press, 2001

8. Swerdlow $\mathrm{SH}$, Campo $\mathrm{E}$, Harris $\mathrm{NL}$, et al: WHO Classification of Tumours of Haematopoietic and Lymphoid Tissues. Lyon, France, IARC Press, 2008

9. Thiele J, Kvasnicka HM, Facchetti F, et al: European consensus on grading bone marrow fibrosis and assessment of cellularity. Haematologica 90:1128-1132, 2005

10. Tefferi A, Lasho TL, Huang J, et al: Low JAK2V617F allele burden in primary myelofibrosis, compared to either a higher allele burden or unmutated status, is associated with inferior overall and leukemia-free survival. Leukemia 22:756-761, 2008

11. Guglielmelli P, Barosi G, Specchia G, et al: Identification of patients with poorer survival in primary myelofibrosis based on the burden of JAK2V617F mutated allele. Blood 114:1477-1483, 2009

12. Passamonti F, Rumi E, Pietra D, et al: A prospective study of 338 patients with polycythemia vera: The impact of JAK2 (V617F) allele burden and leukocytosis on fibrotic or leukemic disease transformation and vascular complications. Leukemia 24: 1574-1579, 2010

13. Thiele J, Kvasnicka HM, Vardiman JW, et al: Bone marrow fibrosis and diagnosis of essential thrombocythemia. J Clin Oncol 27:e220-e221, 2009; author reply e222-e223

14. Wilkins BS, Erber WN, Bareford $D$, et al: Bone marrow pathology in essential thrombocythemia: Interobserver reliability and utility for identifying disease subtypes. Blood 111:60-70, 2008

15. Brousseau M, Parot-Schinkel E, Moles MP, et al: Practical application and clinical impact of the WHO histopathological criteria on bone marrow biopsy for the diagnosis of essential thrombocythemia versus prefibrotic primary myelofibrosis. Histopathology 56:758-767, 2010

16. Florena $A M$, Tripodo $C$, lannitto $E$, et al: Value of bone marrow biopsy in the diagnosis of essential thrombocythemia. Haematologica 89:911-919, 2004

17. Kreft $A$, Büche $G$, Ghalibafian $M$, et al: The incidence of myelofibrosis in essential thrombocythaemia, polycythaemia vera and chronic idiopathic myelofibrosis: A retrospective evaluation of sequential bone marrow biopsies. Acta Haematol 113:137143, 2005

18. Gianelli U, Vener $C$, Raviele PR, et al: Essential thrombocythemia or chronic idiopathic myelofibrosis? A single-center study based on hematopoietic bone marrow histology. Leuk Lymphoma 47:17741781, 2006

19. Vener C, Fracchiolla NS, Gianelli U, et al: Prognostic implications of the European consensus for grading of bone marrow fibrosis in chronic idiopathic myelofibrosis. Blood 111:1862-1865, 2008

20. Harrison CN, Campbell PJ, Buck G, et al: Hydroxyurea compared with anagrelide in high-risk essential thrombocythemia. N Engl J Med 353:3345, 2005

21. Rüdiger $T$, Weisenburger $D D$, Anderson JR, et al: Peripheral T-cell lymphoma (excluding anaplastic large-cell lymphoma): Results from the NonHodgkin's Lymphoma Classification Project. Ann Oncol 13:140-149, 2002

22. Lones MA, Auperin A, Raphael $M$, et al: Mature B-cell lymphoma/leukemia in children and adolescents: Intergroup pathologist consensus with the revised European-American Lymphoma Classification. Ann Oncol 11:47-51, 2000

23. Thiele J, Kvasnicka HM: Chronic myeloproliferative disorders with thrombocythemia: A comparative study of two classification systems (PVSG,
WHO) on 839 patients. Ann Hematol 82:148-152, 2003

24. Hussein $K$, Bock $O$, Theophile $K$, et al: JAK2 (V617F) allele burden discriminates essential thrombocythemia from a subset of prefibrotic-stage primary myelofibrosis. Exp Hematol 37:1186-1193.e7, 2009

25. Girodon F, Dutrillaux F, Broséus $J$, et al: Leukocytosis is associated with poor survival but not with increased risk of thrombosis in essential thrombocythemia: A population-based study of 311 patients. Leukemia 24:900-903, 2010

26. Chim CS, Kwong YL, Lie AK, et al: Long-term outcome of 231 patients with essential thrombocythemia: Prognostic factors for thrombosis, bleeding, myelofibrosis, and leukemia. Arch Intern Med 165: 2651-2658, 2005

27. Cervantes F, Alvarez-Larrán A, Talarn $C$, et al: Myelofibrosis with myeloid metaplasia following essential thrombocythaemia: Actuarial probability, presenting characteristics and evolution in a series of 195 patients. Br J Haematol 118:786-790, 2002

28. Passamonti $F$, Rumi $E$, Arcaini $L$, et al: Prognostic factors for thrombosis, myelofibrosis, and leukemia in essential thrombocythemia: A study of 605 patients. Haematologica 93:16451651, 2008

29. Wolanskyj AP, Schwager SM, McClure RF, et al: Essential thrombocythemia beyond the first decade: Life expectancy, long-term complication rates, and prognostic factors. Mayo Clin Proc 81:159-166, 2006

30. Gangat N, Wolanskyj AP, McClure RF, et al: Risk stratification for survival and leukemic transformation in essential thrombocythemia: A single institutional study of 605 patients. Leukemia 21: 270-276, 2007

31. Passamonti F, Rumi E, Pungolino $E$, et al: Life expectancy and prognostic factors for survival in patients with polycythemia vera and essential thrombocythemia. Am J Med 117:755-761, 2004

32. Alvarez-Larrán A, Cervantes F, Bellosillo B, et al: Essential thrombocythemia in young individuals: Frequency and risk factors for vascular events and evolution to myelofibrosis in 126 patients. Leukemia 21:1218-1223, 2007

33. Barbui T, Barosi G, Birgegard G, et al: Philadelphia-negative classical myeloproliferative neoplasms: Critical concepts and management recommendations from European LeukemiaNet. J Clin Oncol 29:761-770, 2011 